\title{
iBE: A Novel Bandwidth Estimation Algorithm for Multimedia Services over IEEE 802.11 Wireless Networks
}

\author{
Zhenhui Yuan, Hrishikesh Venkataraman, and Gabriel-Miro Muntean \\ Performance Engineering Laboratory, School of Electronic Engineering \\ Dublin City University, Ireland \\ \{yuanzh, hrishikesh, munteang\}@eeng.dcu.ie \\ http://elm.eeng.dcu.ie/ munteang/, \\ http://elm.eeng.dcu.ie/ hrishikesh/HomePage.htm
}

\begin{abstract}
Recently, multimedia streaming services over IEEE 802.11 based wireless networks have increased dramatically. This results in manifold increase in the bandwidth requirement, especially for high-quality multimedia services. Given the bandwidth constraint in the wireless networks, one of the most critical factors in improving the end-to-end performance of multimedia application is the fast and accurate estimation of bandwidth. This paper proposes a novel bandwidth estimation algorithm, iBE. The significant feature of iBE is that it relies on multimedia packets only from the application layer. In addition, iBE recognizes the dynamic fluctuations of the wireless channel quickly, which in-turn enables iBE to be used for real-time services. The experimental results demonstrate that the accuracy of the bandwidth estimated by iBE is significantly superior to other methods like Spruce. Secondly, even in high traffic conditions, the bandwidth estimated by iBE is very close to the actual measured bandwidth, unlike the other state-of-the-art methods.
\end{abstract}

Keywords: Wireless networks, bandwidth estimation, multimedia service.

\section{Introduction}

The popularity of multimedia-based services such as live multimedia streaming, Video-on-demand (VoD), IPTV, etc in the wireless networks has been exponentially increasing. In case of a wired network, the network capacity and the bandwidth is not a major constraint [1]. However, in a wireless network with continuous and dynamic fluctuations in the wireless channel, there are several challenges in case of streaming video and other data traffics [2]. Multimedia streams require large amount of bandwidth which is usually not freely available. Hence, the wireless network operators are greedy for more bandwidth, in order to simultaneously serve voice and video traffic to a large umber of users [3]. Unfortunately, with an increasing number of users in the wireless network, the quality of the multimedia applications fluctuates rapidly due to the decrease in

T. Pfeifer and P. Bellavista (Eds.): MMNS 2009, LNCS 5842, pp. 6\$-80, 2009.

(C) IFIP International Federation for Information Processing 2009 
the per-user network capacity. In addition, the mobility of the user [4], dynamic fluctuation in the wireless channel [5] and cross traffics [6] also deteriorates the quality of the multimedia services.

Previous works such as "WBest" [7], [8] and "Spruce" [9] provides somewhat efficient solution to estimate bandwidth in the network. However, these methods rely on additional probing traffics which create a negative influence on the realtime service due to the introduction of extra bandwidth cost. This in-turn results in the end users having to pay additional cost just for estimating the bandwidth of the network. Demircin and Van Beek proposed a new on-line applicationlayer bandwidth measurement method [10] based on block-ACK mechanism of 802.11e. However, 802.11e protocol has been only used in the recent years and it increases the complexity of the original 802.11 MAC architecture (like $802.11 \mathrm{~b} / \mathrm{g}$ ) which is more popular in current commercial market. Additionally, IEEE 802.11e increases the implementation cost and the real-time constraints have become a lot tighter [11].

This paper proposes a novel intelligent bandwidth estimation algorithm (iBE) for multimedia delivery over IEEE 802.11 wireless networks. iBE makes use of the information related to multimedia packets delivery at the application layer only. iBE estimates the bandwidth from the data packets transmitted over the wireless network; and does not utilize any resource for itself. Hence, iBE estimates the bandwidth very accurately. Apart from estimation accuracy, another major benefit of iBE is that it leads to a simpler implementation and lower computation than the other state-of-the-art methods like Spruce and WBest.

The paper is organized as follows. Section 2 shows the related work in bandwidth estimation methods. In Section 3, detailed description of iBE is provided. Section 4 and Section 5 presents the simulation setup and the result analysis respectively. Finally, Section 6 concludes the paper.

\section{Related Work}

Recently, bandwidth estimation techniques have drawn widespread interests in network management arena. Current research on bandwidth estimation algorithms could be classified into three categories [9], [12]: packet dispersion measurement (PDM), probe gap model (PGM) and probe rate model (PRM). The PDM techniques, such as the packet pair or packet train, estimates network capacity by recording the packet inter-arrival time. Extensive research works have been carried out based on the PDM technique, and several bandwidth estimation techniques have been proposed - "Nettimer" [13], and "bprobe" [14]. However, the main disadvantage of PDM-based technique is that they have very low accuracy when applied to the wireless networks.

Commonly used bandwidth estimation techniques like "Spruce" and the recently proposed "IGI" [15] are based on PGM. The basic principle of PGM is that the server sends a probe packet pair with time dispersion, $T_{\text {in }}$, and after successful transmission, the receiver records a changed dispersion time, $T_{\text {out }}$. The value, $T_{\text {out }}-T_{\text {in }}$ would be the time for transmitting cross traffics under the 
condition that a single bottleneck link is assumed. The cross traffic rate, $\mathrm{BW}_{\mathrm{c}}$, could be written as $\mathrm{BW}_{\mathrm{c}}=\left(\mathrm{T}_{\text {out }}-\mathrm{T}_{\text {in }}\right) \times \mathrm{C} / \mathrm{T}_{\mathrm{in}}$, where $C$ is the capacity of the network. Hence, the estimated available bandwidth would be $C-\mathrm{BW}_{\mathrm{c}}$. However, the main disadvantage of PGM is that it assumes that the network capacity is known. Hence, a faster estimation could be done along with an increase in the accuracy of estimation. In reality, however, the network capacity is not always known beforehand.

The PRM techniques such as "pathChirp" [16] and "pathload" [17], estimate bandwidth using three kinds of traffic rates: sender-side probing rate $\left(C_{s}\right)$, receiver-side probing rate $\left(C_{\mathrm{r}}\right)$ and available bandwidth $(\mathrm{BW})$. If it is considered that $C_{\mathrm{s}}$ gets increased to a level bigger than $(\mathrm{BW})$, then $C_{s}$ would exceed $C_{\mathrm{r}}$ as a result of packet delay at bottleneck link due to queuing mechanism inside routers. Hence, it is critical to find out such level at which $C_{\mathrm{s}}$ starts to become bigger than $C_{\mathrm{r}}$, and this $C_{\mathrm{s}}$ would be measured as available bandwidth.

\section{The Novel Intelligent Bandwidth Estimation Algorithm}

\subsection{Background}

The basic idea of iBE is to make use the difference between the packet's transmission time and reception time at MAC layer. In IEEE 802.11 b MAC protocol [18], the receiver sends acknowledge (ACK) packet for each frame successfully transmitted as shown in Fig. 1.

The RTS/CTS mechanism of MAC layer reduces frame collisions brought by the hidden terminal problem [19]. Even though RTS/CTS packets introduce additional overhead, the throughput loss introduced by RTS/CTS is less due to the smaller packet size (44 bytes and 38 bytes in our simulation) as compared to multimedia packets in the application layer. Inter-frame spacing (IFS) in MAC mechanism reduces the probability of conflict among different packets, thus proving an efficient use of wireless bandwidth. In addition, it should be noted that the control signals (RTS, CTS and ACK) and IFS (SIFS, SIFS) take some amount of bandwidth resources.

The key principle of iBE is to efficiently use packet delay during transmission. Since the waiting time in MAC buffer doesn't constitute the actual bandwidth, as shown in Fig. 1, only the delay in transmitting raw multimedia data is considered. An illustration of various types of delay is shown in Fig. 2.

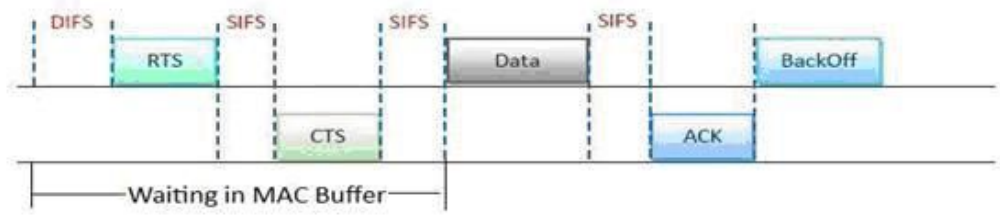

Fig. 1. Packet Sequence in 802.11b MAC Layer 


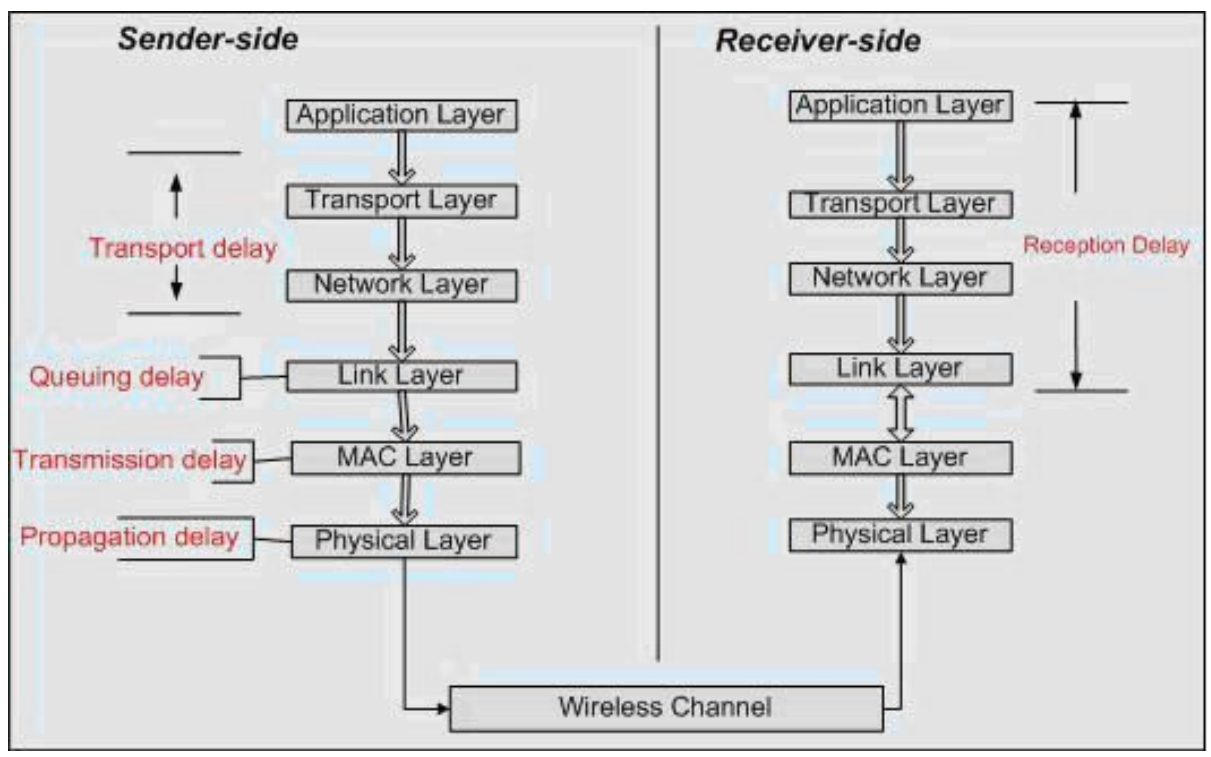

Fig. 2. Types of Packet Delay

Transport delay exists both at sender and receiver side. It is the delay incurred due to reassembling of an incoming or outgoing packet that would be transferred to lower layer (MAC) at sender side and higher layer (application) at receiver side. Transport delay is determined by system processing capability which is independent of wireless bandwidth. Another type of delay is reception delay which is the time taken for receiving a packet. Queuing delay indicates how long a packet have to wait in queues (IFQ) until it gets access to the wireless channel and transmission delay is the delay at the physical layer due to the bit by bit transmission of the packet by the sender. The transmission delay is a function of the packet's length and the transmission rate of link. Finally, the propagation delay is the time taken by one binary bit in a packet traveling the wireless link from sender to receiver. It is deterministic and depends on the distance between the sender and the receiver as well as medium of the link (such as fiber optics, wireless link, etc).

\subsection{Overview}

In order to get a more realistic bandwidth, iBE puts time stamps at MAC layer to calculate bandwidth; since packets buffering time (Queuing delay) and processing delay in upper layers do not reflect the wireless bandwidth [5]. A burst of multimedia packets is chosen as a sample $\left(S_{i}\right)$, where $i$ implies the picked sample. The sample size could be computed by:

$$
S_{i}=\text { packet_recvd }_{i} \times P S_{i}
$$


where packet_recvd $_{i}$ is the number of multimedia packets received within a sample at client MAC layer. $P S_{\mathrm{i}}$ is the size of multimedia packet with MAC header. The time taken to transmit application data $\left(T_{i}\right)$ is calculated as follows:

$$
\begin{aligned}
& T_{i}=\text { recv_time }_{i}-S_{\_} \text {time }_{i}-\left(\text { packet_recvd }_{i}-1\right) \\
& \times\left(3 \times S I F S+D I F S+\text { Backoff }_{i}+T_{\mathrm{ACK}}+T_{\mathrm{RTS}}+T_{\mathrm{CTS}}\right)
\end{aligned}
$$

In eqn. (2) above, the recv_time $e_{i}$ and $S_{-}$time $_{i}$ imply the received time of last packet and the transmission time of first packet of sample $e_{\mathrm{i}}$ respectively. As discussed before, the time taken in MAC buffer such as SIFS, DIFS, TACK, TRTS and TCTS are subtracted from sample transmission time. Here $T_{\mathrm{ACK}}$, $T_{\mathrm{RTS}}$ and $T_{\mathrm{CTS}}$ are time cost for transmitting ACK, RTS and CTS packets. Similarly the Backof $f_{i}$, which means the back off time between two consecutive packets, is subtracted. Back off time depends on current contention window size. Now, the instant bandwidth (instantBW) for a sample is calculated every $5 \mathrm{~ms}$ according to the equation(3):

$$
\text { instant } B W=S_{i} / T_{i}
$$

It should be noted that the time space for periodic bandwidth estimation is chosen as $5 \mathrm{~ms}$. The primary reason for selecting this value is to have a time space that is twice as the frame duration. A standard time frame chosen in the wireless systems (eg: $3 \mathrm{G}$ network like UMTS) is $10 \mathrm{~ms}$. Hence, the $5 \mathrm{~ms}$ time space ensures that bandwidth changes every half the frame duration are accurately estimated. The instant bandwidth estimated is then sent to server as feedback indicating the current network condition.

\section{Experimental Setup}

iBE is modeled and evaluated using Network Simulator 2 (NS-2) version 2.29. Fig. 3 shows the simulation topology where servers send multimedia and cross traffic to clients via a wired network as well as a last hop wireless LAN (WLAN). Multimedia and background traffic share the bottleneck from the access point (AP) to the wireless clients.

Table 1 summarizes the NS-2 configuration used in our experiment. Two additional wireless update patches are deployed in the set-up: NOAH and Marco Fiero patch. NOAH (No Ad-Hoc) is used for simulating the infrastructure WLAN environment whereas Marco Fiero's patch provides a more realistic wireless network environment. As a result, in our experiment, we consider four bandwidth levels: 1, 2, 5.5 and $11 \mathrm{Mbps}$ depending on the distance of the wireless devices from the AP. Fig. 4 shows the characteristic of the actual IEEE 802.11b network. The area with the dark color in Fig. 4 represents higher bandwidth than that with light color. For instance, the rate in the center area is $11 \mathrm{Mbps}$, while the outside area is only $1 \mathrm{Mbps}$. 


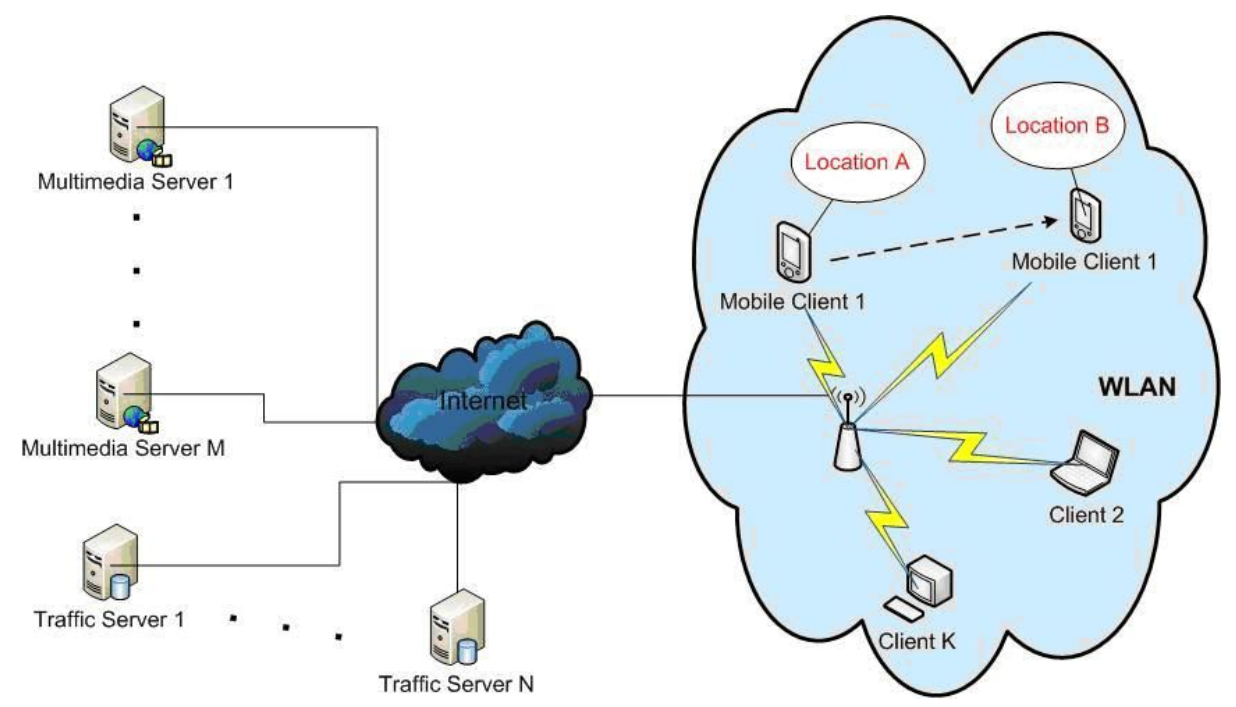

Fig. 3. Simulation Network Topology

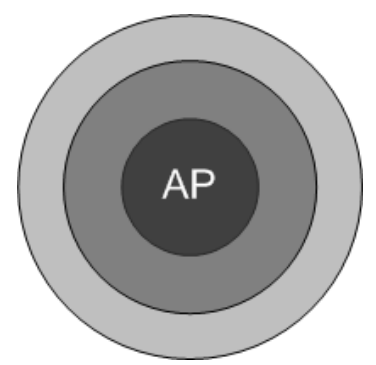

Fig. 4. Dynamic Bandwidth around Access Point (IEEE 802.11b)

$W_{\min }$ and $W_{\max }$ are the minimum and maximum values of contention window. Basic rate, sending rate of control packets (ACK, RTS, CTS), is set as $1 \mathrm{Mbps}$.

In our experiment, six separate tests were conducted for estimating the bandwidth over different network conditions. Each test consisted of one to three unicast video traffics. The client were designed to move from $t=5 \mathrm{~s}$ at the speed of $1 \mathrm{~m} / \mathrm{s}$. Variable network conditions were also realized by varying current traffic loads. This was done by generating CBR/UDP cross traffic using 1500 bytes packets. Additionally, the number of video traffic was scheduled to increase with each test. It should be noted that with an increase in the traffic load, the network becomes quite increasingly congested. The main aim of performing these experiments is to verify how iBE works under heavy and increasing traffic load. 
Table 1. Simulation Setup in NS-2.29

\begin{tabular}{|l|l|}
\hline Parameter & Description of Parameter \\
\hline Transport Protocol & UDP \\
\hline Wireless protocol & $802.11 \mathrm{~b}$ \\
\hline Routing protocol & NOAH \\
\hline Error Model & Marco Fiero patch \\
\hline Wired Bandwidth & 100 Mbps LAN \\
\hline MAC header & 52 bytes \\
\hline Wmin & 31 \\
\hline Wmax & 1023 \\
\hline ACK & 38 bytes \\
\hline CTS & 38 bytes \\
\hline RTS & 44 bytes \\
\hline SIFS & $10^{-6}$ sec \\
\hline DIFS & $50^{-6}$ sec \\
\hline Basic rate & 1 Mbps \\
\hline
\end{tabular}

\section{Experimental Tests and Results}

The performance study compares the measured bandwidth and estimated bandwidth (iBE and Spruce). The measured bandwidth is the actual bandwidth of the network that is measured and it indicates the maximum throughput that an application can obtain. The estimated bandwidth (iBE, Spruce) signifies the maximum end-to-end throughput achieved with cross traffic interferences.

Fig. 5 shows the comparison results of measured bandwidth (calculated from trace results of NS-2) and estimated bandwidth (iBE and Spruce) for periods from 0 to 200 seconds without cross traffic. Two different tests were conducted. The first Test consists of one server and one client using a topology similar to that presented in Fig. 3, whereas, Test two consists of two servers and two clients.

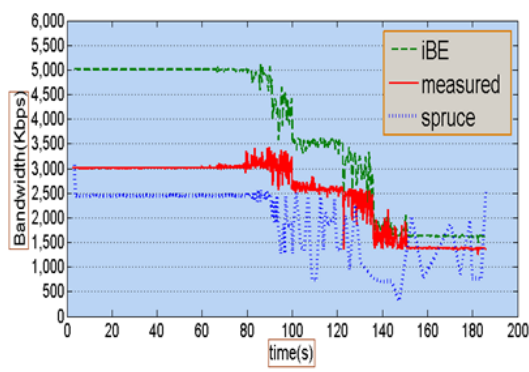

(a)

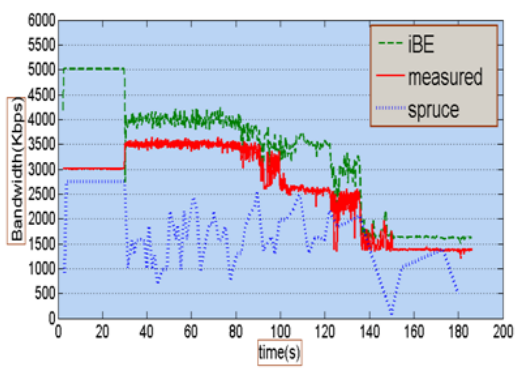

(b)

Fig. 5. Comparison of Estimated and Measured Bandwidth without Cross Traffic 
The results of these two tests are shown in Fig. 5 (a) and Fig. 5 (b) respectively, for both Spruce and iBE. In case of Spruce, the probing traffic used CBR/UDP flow to send packets of 1500 bytes with the rate of $0.15 \mathrm{Mbps}$. In case of iBE however, there is no probing traffic. In addition, the Spruce traffic started at $\mathrm{t}=$ $3 \mathrm{~s}$, whereas that of iBE started at $\mathrm{t}=2 \mathrm{~s}$. A video clip was transmitted to client via high speed (100 Mbps) wired network and IEEE 802.11b WLAN. In case of Test one, as shown in Fig. 5 (a), it was observed that the estimated available bandwidth dropped when the distance between mobile client and AP increased. Also, as shown in Fig. 5 (a), the bandwidth fluctuates at around 80s and 130s due to interference of incoming cross traffic. In order to assess the performance of bandwidth estimation, the concept of average estimated bandwidth has been introduced. The average bandwidth estimated by iBE and Spruce in Test one were 3.52 Mbps and 1.51 Mbps respectively, both of which were notably different from measured bandwidth value of $2.96 \mathrm{Mbps}$. However, there was a difference in the errors - 0.56 and 1.45 for iBE and Spruce respectively. Hence, it could be observed that, on an average, iBE generated fewer errors than Spruce although Spruce performed better in the first 80 s of the simulation scenario.

With the same topology, in Test two, two video clips with the same size were sent to clients using unicast mechanism. The error of iBE and Spruce were 0.29 and 1.63 respectively, demonstrating that iBE outperforms Spruce in heavy traffic condition (two clients). In addition, for both one and two clients, iBE provided smoother estimated bandwidth during stable periods. In Fig. 5 (a) such period

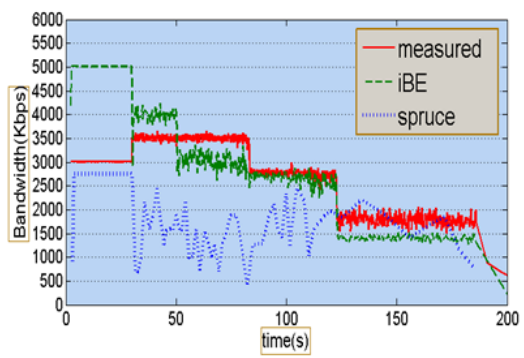

(c)

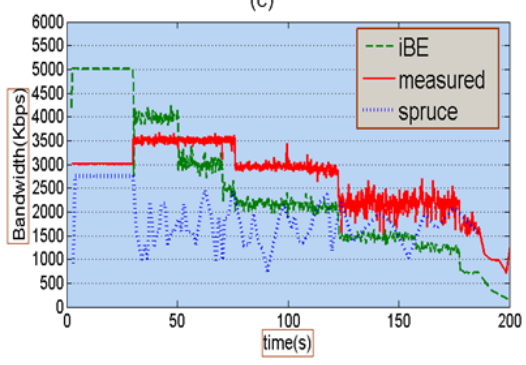

(e)

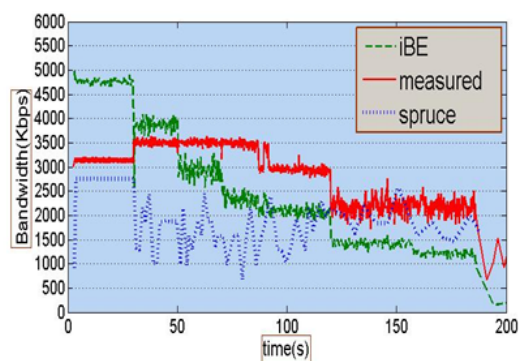

(d)

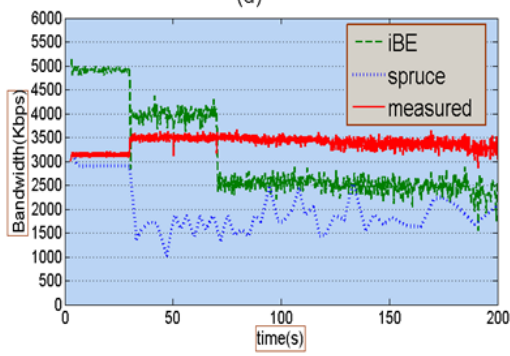

(f)

Fig. 6. Comparison of Estimated and Measured Bandwidth with Cross Traffic 
lasted from 0 to 80s, 100 to 120 s and 130 to 200s while in Fig. 5 (b), it lasted from 30 to 90 s and 100 to 120s. Tests three, four, five and six, involve simulations with participation of constant bit rate over UDP cross traffic of different average bit-rates. This is a significant difference as compared to Test one and Test two. Results of these four tests are shown in Fig. 6. In Test three, two video servers start transmitting at $\mathrm{t}=2 \mathrm{~s}$ and $\mathrm{t}=30 \mathrm{~s}$, and the cross traffic began at $\mathrm{t}=50 \mathrm{~s}$, as shown in Fig. 6 (a). Fig. 6 (b) presents the results of Test four, which involves two video servers transmitting as in test three, but the two cross traffic sources started at $\mathrm{t}=50 \mathrm{~s}$ and $\mathrm{t}=70 \mathrm{~s}$. Fig. 6 (c) illustrates the results of Test five, which involves an additional cross traffic source which starts at $\mathrm{t}=80 \mathrm{~s}$. Test six adds an additional video traffic source to the network, which began delivering video at $\mathrm{t}=50 \mathrm{~s}$. The results are presented in Fig. 6 (d). Overall, it can be observed from Fig. 6 (a) - Fig. 6 (d) that the performance of iBE was significantly closer to the actual measured bandwidth in all the four cases of Tests.

It is clear from the Fig. 5 and Fig. 6 that the bandwidth pattern estimated by $\mathrm{iBE}$ is similar to the actual measured bandwidth, though some delays are encountered in its estimation. This delay arises mainly due to the delay of the multimedia packets. However, it should be noted that the bandwidth estimated by $\mathrm{iBE}$ is always closer to the measured bandwidth than that of Spruce. Fig. 7 shows the average bandwidth for the six experiments (six Tests) as shown in Table 2. Table 2 itemizes the six experimental tests as well as the average bandwidth based on iBE, Spruce and actual measured bandwidth. The error columns in Table 2 show the difference between the measured bandwidth and estimated bandwidth (iBE and Spruce). For instance, in Test four, bandwidth error of iBE is $0.38 \mathrm{Mbps}$ whereas that of Spruce is $1.05 \mathrm{Mbps}$, i.e., $\mathrm{iBE}$ is $63.8 \%$ better than Spruce. Additionally, the bandwidth error of iBE are 0.25 Mbps and 0.14 Mbps for Test five and Test six respectively, indicating $34.2 \%$ and $63.1 \%$ improvement when traffic load became heavier.

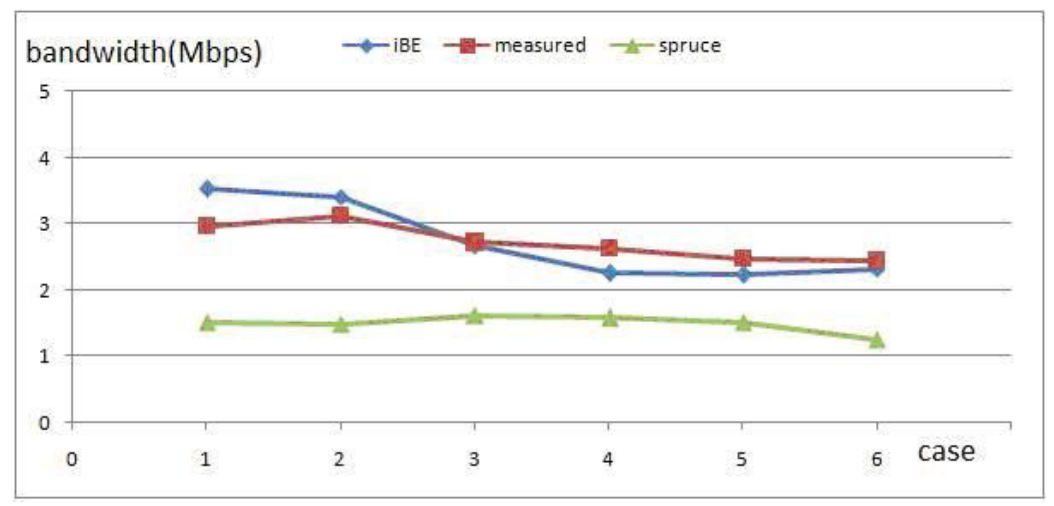

Fig. 7. Average Bandwidth based on Estimated and Measured Values 
Table 2. Bandwidth Estimation for the Six Experiments

\begin{tabular}{|c|c|c|c|c|c|c|c|}
\hline $\begin{array}{l}\text { Sr. } \\
\text { No. }\end{array}$ & \begin{tabular}{|c|} 
Video \\
Clients
\end{tabular} & \begin{tabular}{|c|} 
Cross \\
Traffic \\
CBR/UDP
\end{tabular} & $\begin{array}{c}\text { Measured } \\
\text { BW } \\
\text { (Mbps) }\end{array}$ & $\begin{array}{c}\text { Estimated } \\
\text { BW } \\
i B E \\
(\mathrm{Mbps})\end{array}$ & $\begin{array}{c}\text { Estimated } \\
\text { BW } \\
\text { Spruce } \\
\text { (Mbps) }\end{array}$ & $\begin{array}{c}\text { BW Error } \\
\text { Measured } \\
\& \text { iBE } \\
\text { (Mbps) }\end{array}$ & \begin{tabular}{|c|} 
BW Error \\
Measured \\
\& Spruce \\
(Mbps)
\end{tabular} \\
\hline 1 & 1 & None & 2.96 & 3.52 & 1.51 & 0.56 & 1.45 \\
\hline 2 & 2 & None & 3.12 & 3.41 & 1.49 & 0.29 & 1.63 \\
\hline 3 & 2 & $\begin{array}{l}0.5 \mathrm{Mb} / \mathrm{s} \\
1.0 \mathrm{Mb} / \mathrm{s}\end{array}$ & 2.72 & 2.67 & 1.62 & 0.05 & 1.1 \\
\hline 4 & 2 & $\begin{array}{l}0.5 \mathrm{Mb} / \mathrm{s} \\
1.0 \mathrm{Mb} / \mathrm{s}\end{array}$ & 2.63 & 2.25 & 1.58 & 0.38 & 1.05 \\
\hline 5 & 3 & $\begin{array}{l}0.5 \mathrm{Mb} / \mathrm{s} \\
0.5 \mathrm{Mb} / \mathrm{s} \\
1.0 \mathrm{Mb} / \mathrm{s}\end{array}$ & 2.48 & 2.23 & 1.51 & 0.25 & 0.97 \\
\hline 6 & 3 & $\begin{array}{l}1.0 \mathrm{Mb} / \mathrm{s} \\
1.0 \mathrm{Mb} / \mathrm{s} \\
1.0 \mathrm{Mb} / \mathrm{s}\end{array}$ & 2.45 & 2.31 & 1.26 & 0.14 & 1.19 \\
\hline
\end{tabular}

\section{Conclusion and Future Work}

This paper proposes a new intelligent bandwidth estimation algorithm (iBE) for multimedia delivery over wireless networks. The major benefit of iBE is that it uses multimedia application packets instead of extra probing traffic. This results in a much higher accuracy of the estimated bandwidth, as compared to the other state-of-the-art bandwidth estimation methods. In addition to accuracy, iBE provides a quick response, and at the same time, a smoother result with less variation in the estimated bandwidth. This is extremely beneficial for estimating the bandwidth real-time; which could be subsequently used for efficient resource allocation in dynamically changing wireless networks. The future work would focus on improving the accuracy of iBE and deriving mathematical models for achieving this higher accuracy. In addition, the performance of iBE would be verified under different network conditions and different wireless standards like IEEE $802.11 \mathrm{e} / \mathrm{g}$. It is anticipated that such an accurate estimation of the rapidly changing wireless bandwidth, would enable the network operators to use dynamic rate adaptive solutions for multimedia services in a much better fashion. This would in-turn enable high quality of experience for multimedia transmission in the next generation wireless networks.

\section{Acknowledgments}

The authors would like to thank China Scholarship Council and Irish research Council for Science Engineering and Technology (IRCSET) for their support. 


\section{References}

1. Ngo, C.: A Service-oriented Wireless Home Network. In: IEEE Consumer Communications and Networking Conference, Las Vegas, Nevada, USA (January 2004)

2. Venkataraman, H., Haas, H., Yun, S., Lee, Y., McLaughlin, S.: Performance Analysis of Hybrid Cellular Networks. In: IEEE International Symposium on Personal Indoor Mobile Radio Communications (PIMRC), Berlin, Germany (September 2005)

3. Venkataraman, H., Sinanovic, S., Haas, H.: Cluster-based Two-Hop Cellular Networks. International Journal of Communications, Networks and Systems (IJCNS) 1(4), 370-385 (2008)

4. Mercado, A., Liu, K.J.R.: Adaptive QoS for Mobile Multimedia Services over Wireless Networks. In: IEEE International Conference on Multimedia and Expo, NY, USA, July 30 - August 2 (2000)

5. Muntean, G.M., Cranley, N.: Resource Efficient Quality-Oriented Wireless Broadcasting of Adaptive Multimedia Content. IEEE Transactions on Broadcasting 53(1), 362-368 (2007)

6. Robinson, B.P., Liberatore, V.: On the Impact of Bursty Cross-traffic on Distributed Real-time Process Control. In: IEEE International Workshop on Factory Communication Systems, Vienna, Austria (September 2004)

7. Li, M., Claypool, M., Kinicki, R.: WBest: A Bandwidth Estimation Tool for IEEE 802.11 Wireless Networks. In: 33rd Annual IEEE Conference on Local Computer Networks (LCN), Montreal, Canada (October 2008)

8. Li, M., Claypool, M., Kinicki, R.: Packet Dispersion in IEEE802.11 Wireless Networks. In: 2nd IEEE International Workshop on Performance and Management of Wireless and Mobile Networks, Tampa, FL, USA (November 2006)

9. Strauss, J., Katabi, D., Kaashoek, F.: A Measurement Study of Available Bandwidth Estimation Tools. In: The 3rd ACM SIGCOMM conference on Internet measurement, Miami Beach, FL, USA (October 2003)

10. Demircin, M.U., Van Beek, P.: Bandwidth Estimation and Robust Video Streaming Over 802.11E Wireless Lans. In: IEEE International Conference on Multimedia and Expo., Amsterdam, The Netherlands (July 2005)

11. Chung, S., Piechota, K.: Understanding the MAC Impact of 802.11e: Part 2, Silicon and Software Systems, http://www . commsdesign . com/showArticle.jhtml?articleID=16502136

12. Prasad, R.S., Murry, M., Dovrolis, C., Claffy, K.: Bandwidth Estimation: Metrics, Measurement Techniques, and Tools. IEEE Network 17(6), 27-35 (2003)

13. Lai, K., Baker, M.: Nettimer: A Tool for Measuring Bottleneck Link Bandwidth. In: USENIX Symposium on Internet Technologies and Systems, San Francisco, CA (March 2001)

14. Carter, R.L., Crovella, M.E.: Measuring Bottleneck Link Speed in Packet-switched Networks. Performace Evaluation 27-28(8), 297-318 (1996)

15. Hu, N., Steenkiste, P.: Evaluation and Characterization of Available Bandwidth Probing Techniques. IEEE journal on Selected Areas in Communications 21(6) (August 2003)

16. Ribeiro, V., Riedi, R., Baraniuk, R., Navratil, J., Cottrell, L.: Pathchirp: Efficient Available Bandwidth Estimation for Network Paths. In: Passive and Active Measurement Workshop, La Jolla, CA, USA (April 2003) 
17. Jain, M., Dovrolis, C.: End-to-end Available Bandwidth: Measurement Methodology, Dynamics, and Relation with TCP Throughput. IEEE/ACM Transactions on Networking 11(4), 537-549 (2003)

18. IEEE 802.11, 1999 Edition (ISO/IEC 8802-11: 1999), IEEE Standards for Information Technology - Telecommunications and Information Exchange between Systems - Local and Metropolitan Area Network - Specific Requirements - Part 11: Wireless LAN Medium Access Control (MAC) and Physical Layer (PHY) Specifications (1999)

19. Xu, K., Gerla, M., Bae, S.: How Effective is the IEEE 802.11 RTS/CTS Handshake in Ad hoc Networks. In: IEEE Global Telecommunications Conference, Taiwan, China (November 2002) 